\title{
ATUAÇÃO DO ENFERMEIRO NA SAÚDE DO IDOSO ACOMETIDO PELA DOENÇA DE ALZHEIMER
}

\author{
Leonilda dos Santos Pinho ${ }^{1}$, Chennyfer Dobbins Abi Rached ${ }^{2}$
}

1 Graduanda de Enfermagem. Universidade Nove de Julho - UNINOVE. São Paulo - Brasil - Email: São Paulo - Brasil

2 Doutora em Saúde Coletiva; Mestre em Economia da Saúde pela Universidade Federal de São Paulo (UNIFESP); Docente do Programa de Mestrado Profissional em Gestão em Sistemas de Saúde Universidade Nove de Julho - UNINOVE. São Paulo - Brasil email: chennyferr@yahoo.com.br ORCID: https://orcid.org/0000-0002-4499-3716

\section{RESUMO}

A doença de Alzheimer (DA) é uma patologia degenerativa, uma síndrome demencial de curso lento e progressivo, afeta a cognição, comportamento e função física. Acomete principalmente a população idosa, gerando danos a qualidade de vida e sofrimento a pessoa acometida e familiares. O objetivo desse trabalho é descrever os cuidados de enfermagem ao paciente acometido pela Doença de Alzheimer. A pesquisa foi realizada por revisão bibliográfica desenvolvida através de publicações cientificas preferencialmente, no período de 2012 a 2018, utilizando para levantamento dos dados as bases de dados Literatura Latino-Americana e do Caribe em Ciências da Saúde (LILACS) e BDENF do portal da Biblioteca Virtual em Saúde (BVS) e base de dados Scientific Electronic Library Online (SCIELO). Esta revisão permitiu realizar o levantamento dos principais pontos para descrever a assistência de enfermagem a pacientes portadores da doença de Alzheimer, identificando, assim, uma diversidade de problemas decorrentes da doença e cuidados de enfermagem pertinentes.

Palavras chave: Doença de Alzheimer AND, Enfermagem Geriátrica AND, Demência senil tipo Alzheimer AND, Cuidados de enfermagem Alzheimer AND.

\section{ABSTRACT}

Alzheimer's disease (AD) is a degenerative pathology, a dementia syndrome of slow and progressive course, affects cognition, behavior and physical function. It mainly affects the elderly population, causing damage to the quality of life and suffering to the affected person and family members. The aim of this study is to describe the nursing care of patients with Alzheimer's disease. The research was carried out by a bibliographical review developed through scientific publications preferably in the period from 2012 to 2018, using for data collection the Latin American and Caribbean Literature in Health Sciences (LILACS) and BDENF databases of the Library portal 
Virtual Health Library (VHL) and the Scientific Electronic Library Online (SCIELO) database. This review allowed to carry out the survey of the main points to describe the nursing care to patients with Alzheimer's disease, thus identifying a diversity of problems arising from the disease and pertinent nursing care.

Keywords: Alzheimer 's Disease AND, Geriatric Nursing AND, Senile dementia type Alzheimer' s AND, Nursing Care Alzheimer 's AND.

\section{INTRODUÇÃO}

A doença de Alzheimer (DA) é uma patologia degenerativa, uma síndrome demencial de curso lento e progressivo, afeta a cognição, comportamento e função física. Acomete principalmente a população idosa, gerando danos a qualidade de vida e sofrimento a pessoa acometida e familiares ${ }^{1,2}$.

Caracterizada pela perda neuronal, a região temporal medial é a primeira a ser acometida. A DA pode ser decorrente de excesso de beta amilóide, proteína tau hiperfosforizada, "disfunção neurovascular, processos inflamatórios, stress oxidativo e disfunção mitocondrial"3.

Mundialmente, 25 milhões de pessoas são portadoras da DA, e 90\% dos casos acometem idosos acima dos 65 anos de idade. As demências geram um gasto anual de cerca de "315 milhões de dólares", cerca de 17.964 dólares por pessoa acometida $^{1,2}$.

No Brasil, aproximadamente $7,1 \%$ das pessoas acima de 65 anos de idade são portadoras de demências, equivalente a 1,1 milhão de idosos. Destas, 55\% é a DA. No estado de São Paulo a taxa de incidência da DA é de 7,7 para cada mil pessoas ${ }^{1}$. Segundo dados disponibilizados pelo site do DATASUS, no mês de março de 2018 ocorreram 51 internações hospitalares e 3 óbitos declarados decorrentes de complicações de demências. O gasto total foi de $\mathrm{R} \$ 630.907,13$ somente no mês citado no Estado de São Paulo4. 
Inicialmente, a pessoa portadora de DA apresenta episódios de perda de memória e dificuldades para realizar novas tarefas. Ocorre um declínio funcional conforme o avanço da doença. Com o passar do tempo, surgem outras alterações cognitivas, como a diminuição da capacidade de julgamento e realização de cálculos ${ }^{1}$.

Na fase intermediária pode surgir a afasia e apraxia. A pessoa apresenta dificuldades em expressar ideias, surgem alterações no sono e comportamentais como a agressividade e negligência na realização do autocuidado, distúrbios na marcha e redução da força muscular ${ }^{1}$.

$\mathrm{Na}$ fase final, em alguns casos o paciente fica acamado, torna-se totalmente dependente, apresentando incontinência fecal e urinária ${ }^{5}$

Após o início da sintomatologia, a expectativa de vida do paciente é em torno de 7 a $10 \operatorname{anos}^{2}$. Além dos sintomas citados, a pessoa pode apresentar alterações na visão, paladar e olfato 5 .

O principal fator de risco para a pessoa adquirir a DA é o envelhecimento, seguida de gênero feminino, antecedentes familiares, "presença de outros transtornos mentais associados como a Síndrome de Down". Há uma estimativa de que 1 a cada 7 pessoas portadoras da DA vivem sozinhas, aumentando assim, a quantidade de óbitos relacionados as complicações decorrentes da patologiaª ${ }^{2,6}$.

A DA altera o modo de como as atividades de vida diária (AVDs) são realizadas e, dependendo do estágio da demência, a pessoa acometida pode torna-se totalmente dependente de familiares e/ou cuidadores ${ }^{7}$.

\subsection{Problema de pesquisa}

Quais são os cuidados de enfermagem realizados em ambiente domiciliar ao paciente portador da Doença de Alzheimer? 


\section{2 justificativa}

A doença de Alzheimer é um tipo de demência que provoca uma deterioração global, progressiva e irreversível de diversas funções cognitivas (memória, atenção, concentração, linguagem e pensamento e, com o progresso da demência a pessoa acometida necessita de cuidados familiares e/ou de enfermagem. Devido à alta incidência de novos casos da doença na população idosa, se faz necessário realizar revisão bibliográfica para adquirir conhecimentos científicos para garantir uma assistência de Enfermagem de qualidade e eficaz, focada na sintomatologia apresentada pelo paciente portador da DA.

\section{OBJETIVOS}

\subsection{Objetivos gerais}

Buscar evidências científicas para identificar como é realizada a terapêutica, de modo que proporcione alívio aos sintomas existentes, estabilizando-os ou, ao menos permitindo que boa parte dos pacientes tenha uma progressão mais lenta.

Buscar evidências científicas para identificar os principais cuidados de Enfermagem e familiares realizados durante a terapêutica da Doença de Alzheimer.

\subsection{Objetivo específico}

Descrever os cuidados de enfermagem ao paciente acometido pela Doença de Alzheimer. 


\section{METODOLOGIA}

\subsection{Tipo de Estudo}

Tratar-se de uma revisão de literatura, que consiste em um levantamento de referências teóricas já analisadas anteriormente, por procedimento reflexivo, sistemático, controlado e crítico, permitindo a descoberta de novos fatos ou dados, relações ou leis, em qualquer campo de conhecimento. Esta pode ser caracterizada como o primeiro passo do pesquisador para realizar uma pesquisa cientifica; através dela é possível fazer levantamentos dos dados já existentes em material publicado, constituído principalmente por livros-texto, artigos científicos, dissertações, entre outros $^{8}$.

\subsection{Contexto}

A busca de pesquisas para elaboração da revisão integrativa foi realizada em banco de dados de acesso livre, trabalhos publicados em português nas bases de dados Literatura Latino-Americana e do Caribe em Ciências da Saúde (LILACS) e BDENF do portal da Biblioteca Virtual em Saúde (BVS) e base de dados Scientific Electronic Library Online (SCIELO).

Foram utilizados como critério de inclusão os artigos de pesquisas indexados nas bases de dados entre os anos de 2012 até 2018 , no idioma em português que estavam disponibilizados sua versão na íntegra, para a realização da pesquisa os descritores foram utilizados em português.

\subsection{Coleta dos Dados}

A coleta de dados foi realizada pela pesquisadora. Para tanto, foi utilizado um instrumento de coleta de dados que abordará os passos necessários a resposta da 
questão da revisão integrativa, como: título da obra; base de indexação, procedência da publicação; local e ano, objetivos; principais resultados e conclusões.

A questão norteadora desta pesquisa foi: Quais são os cuidados de enfermagem realizados em ambiente domiciliar ao paciente portador da Doença de Alzheimer?

A coleta do material foi feita por meio dos Descritores de Ciências da Saúde (DeCS) uma busca nas bases de dados bibliográficas selecionadas de documentos de pesquisas primárias referente ao tema atuação do enfermeiro na saúde do idoso acometido pela doença de Alzheimer.

Nesta etapa da coleta foram utilizados os descritores: Doença de Alzheimer AND, Enfermagem Geriátrica AND, Demência senil tipo Alzheimer AND, Cuidados de enfermagem Alzheimer AND, consultados por palavra ou termo e por índice permutado. Desta pesquisa foram obtidos os resultados do quadro 1.

\begin{tabular}{|l|c|c|c|l|}
\hline $\begin{array}{l}\text { DeCS/ Bases } \\
\text { de Dados }\end{array}$ & $\begin{array}{l}\text { Doença de } \\
\text { Alzheimer AND }\end{array}$ & $\begin{array}{l}\text { Enfermagem } \\
\text { Geriátrica AND }\end{array}$ & $\begin{array}{l}\text { Demência Senil } \\
\text { Tipo Alzheimer } \\
\text { AND }\end{array}$ & $\begin{array}{l}\text { Cuidados de } \\
\text { enfermagem } \\
\text { Alzheimer AND }\end{array}$ \\
\hline LILACS & 1120 & 457 & 845 & 45 \\
\hline SCIELO & 615 & 240 & 5 & 9 \\
\hline BDENF & 4 & 15 & 3 & 2 \\
\hline
\end{tabular}

Os artigos serão analisados, comparados e observados quanto sua semelhança e/ou divergência para a realização do desenvolvimento do tema.

\section{DESENVOLVIMENTO}

\subsection{Terapêutica farmacológica}

A terapêutica farmacológica proporciona a redução dos sintomas, não há cura para a DA. Tem como objetivo estimular ou manter a capacidade cognitiva e evitar 
anormalidades psicológicas, afim de melhorar a qualidade de vida e estado de saúde da pessoa portadora da $\mathrm{DA}^{5}$.

Inibidores da colinesterase proporcionam melhora o desempenho das funções cognitivas, facilitando a realização das AVDs. Na fase inicial recomenda-se a administração dos fármacos Tacrina (Cognex), Donepezil (Aricept): administrado por via oral, 1 vez ao dia, com a dose recomendada de 5 a 10 mg; Rivastigmina (Exelon): administrado por via oral, duas vezes ao dia, com a dose diária de 6 a 12 mg. e o Galantamina (Razadyne): administrado por via oral, 1 vez ao dia, com a dose recomendada de 16 a $24 \mathrm{mg}^{5,9}$.

$\mathrm{Na}$ fase moderada e avançada, recomenda-se a administração da Memantina (Namenda): administrado por via oral, nas duas primeiras semanas de tratamento a dose única diária é de $5 \mathrm{mg}$. Após o período, administra-se duas doses, não podendo exceder $20 \mathrm{mg}$ diárias ${ }^{5,9}$.

Os neurolépticos são recomendados caso ocorra manifestações como ansiedade, delírios, alucinações, distúrbios do sono, depressão e agitação ${ }^{9}$.

\subsection{Intervenções de enfermagem para estimular a função cognitiva}

O enfermeiro atua estimulando a função cognitiva da pessoa acometida, realiza medidas para a redução do estresse, ansiedade e agitação, estimula o autocuidado, garante a segurança física ${ }^{10}$.

Algumas intervenções terapêuticas podem ser realizadas para a estimulação da função cognitiva, como a reabilitação da memória, neuropsicológica e terapia de reorientação ${ }^{10}$.

Reabilitação da memória: são realizados exercícios de repetição e treino auxiliam na redução de perda de memória. Permite melhora no funcionamento mnésico. O tipo de 
reabilitação varia conforme as necessidades individuais do paciente. Algumas atividades envolvem estímulo visual, sensorial, motores, cinéticos e/ou verbais ${ }^{5}$.

A reabilitação neuropsicológica é um tratamento biopsicossocial, melhora a cognição e comportamentos disfuncionais. Estimula o funcionamento físico, psíquico e social. tem como objetivo reduzir a deteriorização cognitiva. Envolve a residência, familiares e pessoa de convívio, como os cuidadores. Associado a terapêutica farmacológica apresenta bons resultados ${ }^{5}$.

A terapia de orientação para a realidade estimula o paciente a adaptar-se as suas limitações e proporciona melhora da autoestima ${ }^{1}$.

\subsection{Orientações para o paciente, familiares e cuidadores.}

O enfermeiro deve realizar orientações sobre a doença e seu avanço, terapêutica, cuidados com o armazenamento, administração de fármacos, dietoterapia, exercícios físicos conforme tolerância e exercícios cognitivos. Esclarecer dúvidas é fundamental para que não ocorra desgaste físico de emocional das pessoas envolvidas no processo de cuidar ${ }^{10}$.

Os cuidadores e familiares devem ter uma boa comunicação com a pessoa acometida pela doença de Alzheimer para que haja equilíbrio no cuidado prestado ${ }^{10}$.

$\mathrm{Na}$ fase inicial da doença, é necessário proporcionar suporte emocional para a pessoa acometida, pois pode apresentar alterações emocionais e suporte cognitivo devido ao fato de que se inicia a redução da capacidade de julgamento, raciocínio e outras habilidades nessa fase $\mathrm{e}^{11,12}$.

No estágio intermediário é necessário manter os cuidados citados e a pessoa acometida pode apresentar dificuldades para a realização de atividades diárias de vida como tomar banho, escovar os dentes e alimentar-se. Nesta fase é necessário que as pessoas envolvidas nos cuidados auxiliem nas tarefas e na deambulação ${ }^{11,12}$. 
No estágio grave da doença, surge alterações comportamentais, mudanças no ciclo do sono, perda de apetite e ponderal, incontinência urinária e fecal. Geralmente, o idoso torna-se acamado, necessitando de cuidados integrais como estimulação e auxílio da alimentação, cuidados pessoais, mudança de decúbito a cada duas horas para evitar lesões por pressão, administração correta de fármacos, manter o ambiente seguro para evitar lesões e quedas ${ }^{12}$.

\section{CONSIDERAÇÕES FINAIS}

Através de análises realizadas por artigos foi possível realizar o levantamento dos principais pontos para descrever a assistência de enfermagem a pacientes portadores da doença de Alzheimer, identificando, assim, uma diversidade de problemas decorrentes da doença de Alzheimer. É importante realizar os cuidados de enfermagem direcionados e com um referencial teórico para se obter uma assistência qualificada, voltada para os cuidados ao paciente com a patologia apresentada, pois o Alzheimer não afeta somente o aspecto psicológico do paciente, mas engloba também alterações sociais, espirituais, alimentícias e fisiológicas.

\section{REFERÊNCIAS BIBLIOGRÁFICAS}

1. Brasil. Ministério da saúde. Doença de Alzheimer. Protocolo Clínico e Diretrizes Terapêuticas Portaria SAS/MS n 1.298, de 21 de novembro de 2013. Disponível: http://portalarquivos2.saude.gov.br/images/pdf/2014/abril/02/pcdt-doenca-dealzheimer-livro-2013.pdf. [acessado 01 de maio de 2018]

2. Gonçalves EAG, Carmo JS. Diagnóstico da doença de Alzheimer na população brasileira: um levantamento bibliográfico. Rev Psic Saúde. 2012; 4(2): 170-176. Disponível:http://pepsic.bvsalud.org/scielo.php?script=sci_arttext\&pid=S2177-093X 2012000200010. [acessado 01 de maio de 2018] 
3. Ferreira SHJM. Terapêutica farmacológica na Doença de Alzheimer: progressos e esperanças futuras. Rev Arq Med. Univ. Porto. 2012. Disponível: https://repositorioaberto.up.pt/bitstream/10216/72411/2/28937.pdf. [acessado 01 de maio de 2018]

4. Brasil. Ministério da Saúde. DATASUS. Lista Morb CID-10: Demência. Período: Mar/2018. Disponível: http://tabnet.datasus.gov.br/cgi/tabcgi.exe?sih/cnv/nisp.def

5. Oliveira CL, Freitas C, Monteiro MI. A doença de Alzheimer: características, sintomas e intervenções. Rev Psicol PT. 2013: 1-15; Disponível: http://www.psicologia.pt/artigos/textos/A0662.pdf. [acessado 01 de maio de 2018]

6. Nitzsche BO, Moraes HP, Tavares Júnior AR. Doença de Alzheimer: novas diretrizes para o diagnóstico. Rev Med Minas Gerais 2015; 25(2): 237-243. Disponível: http://www.rmmg.org/artigo/detalhes/1780. [acessado 01 de maio de 2018]

7. Talmelli LF, Vale FA, Gratão AC, Kusumota L, Rodrigues RA. Doença de Alzheimer: declínio funcional e estágio da demência. Acta Paul Enferm. 2013; 26(3):219-25. Disponível: http://www.redalyc.org/service/redalyc/downloadPdf/3070/307027354002 16. [acessado 01 de maio de 2018]

8. Gerhardt TE, Silveira DT [organizadores]. Métodos de Pesquisa. P. 37, 90. Porto Alegre: Editora UFRGS, 2009. Disponível: http://zip.net/bpnzXX. [acessado $01 \mathrm{de}$ junho de 2018]

9. Vale FAC, Corrêa Neto Y, Bertolucci PHF, Machado JCB, Silva DJ, Allam N et al. Tratamento da doença de Alzheimer. Dement Neuropsychol. 2011;5(Suppl 1):34-48. Disponível: http://www.redalyc.org/html/3395/339529025005/. [acessado 23 de setembro de 2018]

10. Camacho ACFL, Abreu LTA, Leite BS. Revisão integrativa sobre os cuidados de enfermagem à pessoa com doença de Alzheimer e seus cuidadores. Rev. Pesq. Cuid. Fund. 2013;5(3):186-193. Disponível: http://www.seer.unirio.br/index.php/cuidadofu ndamental/article/view/1731. [acessado 23 de setembro de 2018] 
11. Zidani M, Arcoverde C, Araújo NB, Vasquesi P, Rios A, Laks J. Alterações motoras e funcionais em diferentes estágios da doença de Alzheimer. Rev Psiq Clín. 2012;39(5):161-165. Disponível: http://www.scielo.br/pdf/rpc/v39n5/a03v39n5. [acessado 24 de setembro de 2018]

12. Ilha S, Zamberlan C, Nicola GDO, Araújo AS, Backes DS. Refletindo acerca da doença de Alzheimer no contexto familiar: implicações para a enfermagem. Rev.Enf. Cen. Oeste. Min. 2014;4(1):492-502. Disponível: http://seer.ufsj.edu.br/index.php/ recom/article/view/378. [acessado 24 de setembro de 2018] 